\title{
Bone Metastases in Advanced Prostate Cancer
}

\section{Fred Saad, MD, FRCS}

\author{
Centre Hospitalier de l'Université de Montréal, Hôpital Notre-Dame, Montreal, Canada
}

DOI: 10.17925/OHR.2005.00.00.1V

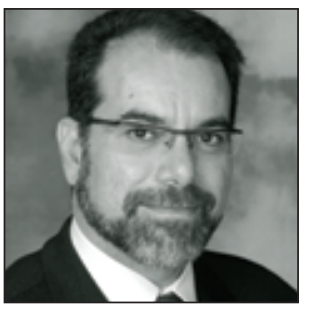

Fred Saad, MD, FRCS, established the first urologic oncology clinic and research center at the University of Montreal, where he is currently Director of Urologic Oncology. In 2004 he was named the first Universtiy of Montreal Chair in Prostate Cancer. Dr Saad is Full Professor of Surgery/Urology at the University of Montreal. He was President of the Quebec Urologic Association 200I-2003. He is

secretary/treasurer of the executive committee of the National Cancer Institute of Canada G-U Group (NCIC-GU) and Canadian Urologic Oncology Group (CUOG), and is a member of the Canadian Prostate Health Council (CPHC). In addition,

he sits on nine editorial boards and serves as a Reviewer for over 20 urology and oncology journals. He has published over 70 articles in peer-reviewed journals and has over 250 published abstracts. He has been an invited speaker/professor over 200 times in 20 different countries.

Dr Saad's main research interests include molecular prognostic markers and hormone-refractory prostate cancer. Currently, he coordinates over 30 clinical and basic research projects in urologic oncology. He obtained his medical degree in 1985 from the University of Montreal and then completed his residency in urology in 1990. He completed a Fellowship in Urologic Oncology in 1992, and became Chief of Urology at Notre-Dame Hospital of the University of Montreal in 1994.

\section{Abstract}

Men with advanced prostate cancer are at high risk for development of bone metastases, resulting in clinically significant skeletal morbidity and severe bone pain. Studies of early-generation bisphosphonates, clodronate, and pamidronate, demonstrated transient palliative effects on bone pain in patients with bone metastases, but failed to demonstrate long-term clinical benefit. A small, open-label study of ibandronate demonstrated significant reductions in pain, but these results have not been confirmed in a larger, randomized, controlled trial. Currently, zoledronic acid is the only bisphosphonate that has demonstrated statistically significant reductions in skeletal morbidity - including durable pain reduction - in this patient population in a randomized placebocontrolled trial. Therefore, zoledronic acid therapy should be considered to prevent skeletal morbidity and improve the quality of life of prostate cancer patients with bone metastases.

\section{Introduction}

Prostate cancer is one of the most common cancers affecting men worldwide. ${ }^{1}$ Current cancer statistics estimate that more than 200,000 men are diagnosed with prostate cancer in the US each year, ${ }^{2}$ and five-year worldwide prevalence is approaching 2.5 million cases. ${ }^{3}$ Approximately $50 \%$ of patients will experience disease recurrence after local therapy consisting of radiation or prostatectomy. ${ }^{4}$ Although advances in the treatment of prostate cancer have extended life expectancy, 65\% to $75 \%$ of patients with advanced disease will develop bone metastases, resulting in accelerated bone resorption and a loss of skeletal integrity that is associated with significant skeletal morbidity, including pathologic fractures, spinal cord compression, and debilitating bone pain. ${ }^{5}$

The incidence of skeletal complications experienced by patients with metastatic prostate cancer is illustrated by the placebo group of a recent study of zoledronic acid in men with hormone-refractory prostate cancer (HRPC) and bone metastases. ${ }^{6,7}$ In this study, nearly 50\% of patients receiving placebo experienced $\geq 1$ skeletal complication and $33 \%$ suffered severe bone pain requiring palliative radiotherapy. A recent study showed that these skeletal complications result in significant decreases in quality-of-life scores. ${ }^{8}$ Therefore, therapies that prevent skeletal complications and reduce bone pain could translate into improvements in quality of life.

Bisphosphonates are potent inhibitors of bone resorption that have demonstrated clinical benefit for the treatment of malignant bone disease and are the standard of care for the prevention of skeletal complications in patients with bone metastases from breast cancer.' Several studies have evaluated the efficacy of bisphosphonates - including etidronate, clodronate, pamidronate, ibandronate, and zoledronic acid - in patients with bone metastases from prostate cancer. However, until the recent trial evaluating the efficacy of zoledronic acid in men with HRPC and bone metastases, no bisphosphonate has demonstrated statistically significant long-term reductions in skeletal morbidity or durable pain reduction in this patient population in a randomized placebo-controlled trial. ${ }^{6}$

Benefit of Bisphosphonates in Advanced Prostate Cancer

\section{Etidronate and Clodronate}

Early-generation bisphosphonates including etidronate and clodronate failed to demonstrate statistically significant, durable clinical benefits in men with bone metastases secondary to prostate cancer. Although single-arm studies of oral and intravenous (IV) clodronate and etidronate demonstrated transient reductions from baseline in pain levels and analgesic use, these decreases did not reach significance. ${ }^{10-13}$ Furthermore, the clinical benefits of these agents have not been demonstrated in randomized controlled trials. ${ }^{14-18}$ In a recent randomized controlled trial involving 209 men with HRPC and bone metastases, IV clodronate $(1,500 \mathrm{mg})$ administered every three weeks in combination with mitoxantrone/prednisone did not significantly improve palliative response (combination of pain and analgesic scores) compared with mitoxantrone/prednisone plus placebo. ${ }^{17}$ Additionally, in a randomized controlled trial involving 311 men with metastatic prostate cancer who were 
receiving first-line hormonal therapy, oral clodronate $(2,080 \mathrm{mg} /$ day $)$ did not significantly extend bone progression-free survival, defined as the time from randomization to the development of symptomatic bone metastases or death from prostate cancer, compared with placebo, after a median of 59 months' follow-up. ${ }^{18}$ Furthermore, the patients receiving oral clodronate experienced significantly more adverse events, particularly gastrointestinal problems, compared with placebo (risk ratio $=1.71 ; \mathrm{p}=0.002$ ).

\section{Pamidronate}

Similarly, randomized studies of pamidronate in men with bone metastases from prostate cancer also failed to demonstrate clinical benefit. ${ }^{19,20}$ In a randomized openlabel trial involving 58 patients, IV pamidronate (60 or $90 \mathrm{mg}$ every two to four weeks) for three months reduced bone pain, but did not consistently reduce biochemical markers of bone turnover. ${ }^{19}$ In a more recent combined analysis of two multicenter, randomized placebo-controlled trials involving 374 patients with HRPC and bone metastases, IV pamidronate (90mg every three weeks) did not significantly reduce brief pain inventory (BPI) pain scores (primary end-point) or analgesic use compared with placebo after 27 weeks of treatment. ${ }^{20}$ Moreover, pamidronate failed to reduce the percentage of patients experiencing a skeletal complication compared with placebo ( $25 \%$ in both treatment groups at 27 weeks).

\section{Ibandronate}

Recently, in a small, open-label study, IV ibandronate demonstrated reduction of bone pain in men with HRPC and bone metastases. ${ }^{21}$ In this trial, 25 patients were treated with ibandronate $(6 \mathrm{mg})$ every four weeks. All patients reported high levels of bone pain at baseline, with a mean pain score of 6.5 on a visual analog scale of $0-10$. After ibandronate treatment, 23 (92\%) patients reported significant reductions from baseline in pain, with a mean pain score of 2.0 $(\mathrm{p}<0.001)$, and nine $(39 \%)$ patients were completely pain-free. In addition, ibandronate significantly reduced analgesic use in $92 \%$ of the patients. However, these results have not been confirmed in a larger randomized controlled study, a particularly important consideration in trials that use a subjective assessment such as pain control given the acknowledged 'placebo effect' that can occur in an open-label trial.

\section{Zoledronic Acid}

In contrast to previous bisphosphonate studies, zoledronic acid has demonstrated objective and durable reductions in skeletal morbidity and pain in patients with prostate cancer and bone metastases. In a large
Figure I: Treatment with Zoledronic Acid Significantly Reduced the Percentage of Patients with $\geq$ I SRE Compared with Placebo Across 24 Months of Treatment in Men with HRPC Metastatic to Bone

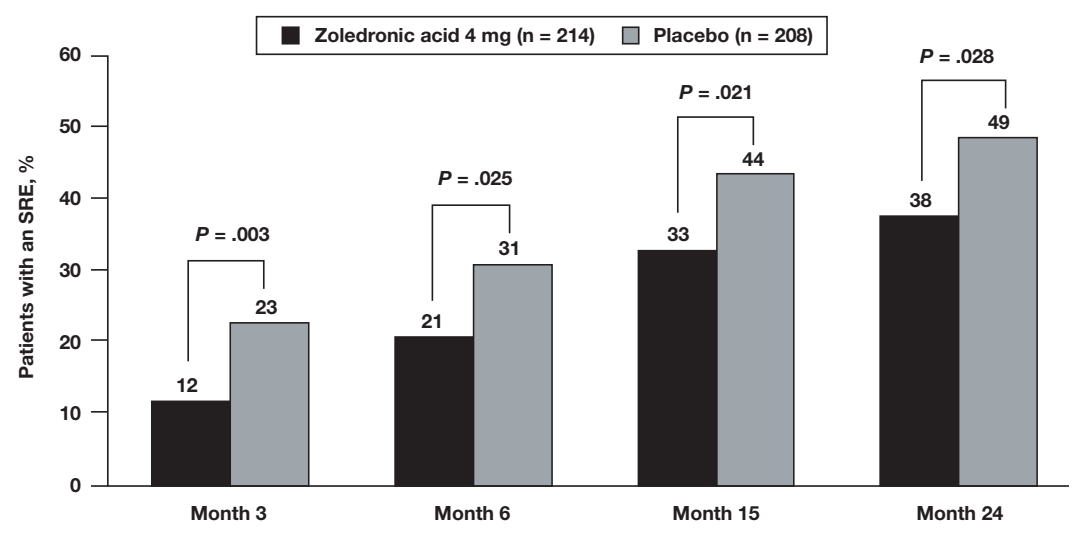

Zoledronic acid (4mg every three weeks for 24 months) significantly reduced the percentage of patients with any SRE compared with placebo at three, six, 15, and 24 months. Data from Saad et al.16,22 and Lipton et al.:24 adapted with permission from Saad et al..$^{25}$

randomized phase III trial, 422 men with HRPC and bone metastases were randomized to zoledronic acid (4mg every three weeks via 15-minute infusion) or placebo for up to 24 months. ${ }^{6,22}$ Efficacy outcomes were assessed by evaluating the development of skeletalrelated events (SREs), defined as a pathologic fracture, spinal cord compression, radiation or surgery to bone, or change of antineoplastic therapy to treat bone pain. This composite end-point provides an objective and comprehensive assessment of treatment effect, on the basis of clinically relevant events. ${ }^{23}$ In addition, bone pain was assessed using the brief pain inventory (BPI) pain scale. Of note, patients who required strong narcotic therapy for pain control were excluded from this study.

Analysis after 24 months of treatment demonstrated that zoledronic acid $(4 \mathrm{mg})$ significantly decreased the percentage of patients with an SRE relative to placebo by $22 \%$ (38\% versus $49 \%$ for placebo; $p=0.028$; see Figure 1). ${ }^{6,22,24,25}$ Moreover, zoledronic acid consistently reduced all types of SREs. ${ }^{7}$ Notably, the clinical benefit of zoledronic acid was apparent early and was maintained throughout the entire course of the trial. Zoledronic acid significantly decreased the percentage of patients who developed an SRE compared with placebo at three months $(\mathrm{p}=0.003)$, at six months $(\mathrm{p}=0.025)$, and at 15 months $(\mathrm{p}=0.021)$ (see Figure 1). ${ }^{6,22,24,25}$ In contrast, treatment with pamidronate for six months demonstrated no benefit compared with placebo in the percentage of patients with an SRE. ${ }^{20}$

After 24 months' follow-up, treatment with zoledronic acid also significantly delayed the median time to first SRE by nearly six months, from 321 days in the placebo 
Figure 2: Treatment with Zoledronic Acid Significantly Increased the Median Time to First SRE Compared with Placebo in Men with HRPC Metastatic to Bone

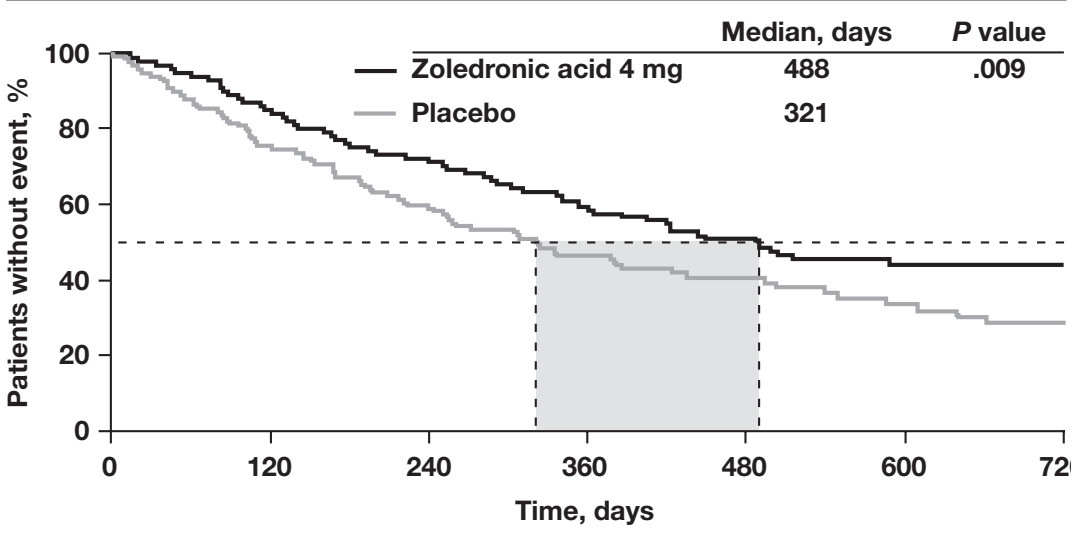

Zoledronic acid (4mg every three weeks for 24 months) significantly delayed the median time to first SRE by more than five months. Shaded area represents difference between medians. Adapted with permission from Saad et al. ${ }^{27}$

Figure 3: Treatment with Zoledronic Acid Significantly Reduced Pain Scores Compared with Placebo in Men with HRPC Metastatic to Bone

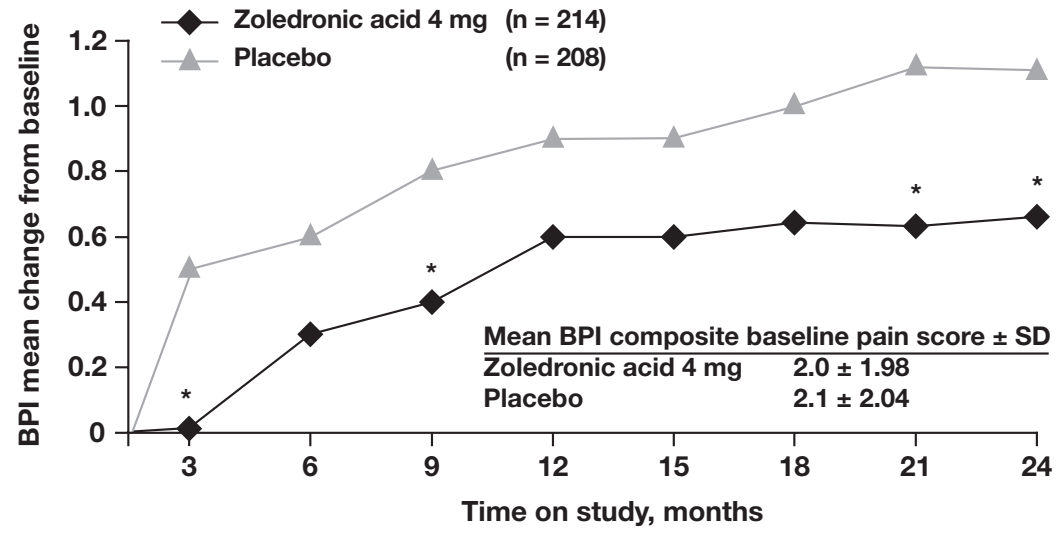

Zoledronic acid (4mg every three weeks for 24 months) consistently reduced BPI pain scores compared with placebo throughout the course of the study. ${ }^{*} p<0.05$. $S D=$ standard deviation. Adapted with permission from Saad et al. ${ }^{27}$

group, to 488 days for patients treated with zoledronic acid ( $p=0.009$; see Figure 2) ${ }^{6,7}$ Additionally, zoledronic acid significantly delayed the median time to first pathologic fracture compared with placebo (median not reached by either treatment group; 25\% quartile $=516$ days for zoledronic acid versus 321 days for placebo; $\mathrm{p}=0.020) .^{7}$ Finally, a planned Andersen-Gill multiple event analysis that took into account both the incidence and timing of skeletal complications demonstrated that zoledronic acid reduced the risk of developing an SRE by $36 \%$ compared with placebo at 24 months (hazard ratio $=0.640 ; \mathrm{p}=0.002) .{ }^{6}$

Retrospective exploratory analyses have further demonstrated that zoledronic acid provided on-going clinical benefit throughout the entire 24-month study period. Second-event analysis showed that zoledronic acid significantly reduced the percentage of patients who developed a second SRE relative to placebo by $32 \%(21 \%$ versus $31 \%$ for placebo; $p=0.017)$, significantly delayed the median time to second SRE (median not reached for zoledronic acid versus 449 days for placebo; $\mathrm{p}=0.006)$, and significantly reduced the risk of developing a second SRE by $40 \%$ (hazard ratio $=0.601 ; \mathrm{p}=0.011$ ) compared with placebo. ${ }^{26}$ These results imply that zoledronic acid has continuing clinical benefit with long-term treatment in this patient population.

In addition to analysis of SREs, BPI composite pain scores were used to assess pain responses in patients receiving zoledronic acid. Patients in both treatment groups began the study with a low mean baseline pain score of approximately two on a scale of $0-10$, and $30 \%$ of patients had no pain at baseline. As expected, pain scores increased from baseline in both treatment groups. However, patients treated with zoledronic acid reported significantly smaller increases in pain scores over the duration of the trial compared with placebo. ${ }^{6}$ Betweengroup differences reached statistical significance at three months $(p=0.003)$, nine months $(p=0.030), 21$ months $(\mathrm{p}=0.014)$, and 24 months $(\mathrm{p}=0.024)$ (see Figure 3$).{ }^{27} \mathrm{In}$ addition, a recent study using survival-adjusted cumulative event analysis demonstrated that zoledronic acid significantly reduced the need for radiation to bone (which can be considered a surrogate for bone pain) by $33.2 \%$ compared with placebo at 24 months $(p=0.034) .{ }^{28}$ Therefore, zoledronic acid offers durable and consistent relief of bone pain in patients with prostate cancer.

Importantly, zoledronic acid demonstrated a favorable overall safety profile. Adverse events were manageable and included mainly the flu-like symptoms common to all IV bisphosphonates (ie, fatigue, anemia, myalgia, and pyrexia); these were observed primarily after the first few infusions. Zoledronic acid also demonstrated an acceptable renal safety profile, with the risk of experiencing an increase in serum creatinine levels similar to that of placebo (hazard ratio $=1.14 ; p=0.752){ }^{7}$

\section{Conclusions}

Skeletal complications resulting from bone metastases can be associated with debilitating bone pain and can negatively affect quality of life in men with advanced prostate cancer. Although several bisphosphonates have been shown to provide transient pain relief for these patients, zoledronic acid is the only bisphosphonate to significantly reduce the incidence, delay the onset of skeletal complications, and provide consistent and durable reduction of pain in a randomized placebo-controlled trial. Therefore, zoledronic acid represents an important advancement in the treatment of bone metastases in patients with 
advanced prostate cancer. Based on this evidence, the 3rd International Consultation on Prostate Cancer developed a prostate cancer treatment algorithm that supports the use of zoledronic acid at first evidence of bone metastases. ${ }^{29}$

The role of bisphosphonate therapy in the treatment of prostate cancer patients continues to evolve. Zoledronic acid has already demonstrated the potential to prevent cancer treatment-induced bone loss and to increase bone mineral density in patients with prostate cancer receiving androgen deprivation therapy. ${ }^{30}$ Furthermore, pre-clinical data suggest that zoledronic acid may potentially prevent bone metastases and delay disease progression. ${ }^{31}$ These studies provide evidence that in the future bisphosphonates will have an important role throughout the treatment continuum for patients with prostate cancer.

References

1. Parkin D M, Pisani P and Ferlay J, "Global cancer statistics", C A Cancer J. Clin. (1999), 49: pp. 33-64.

2. Jemal A, Tiwari R C, Murray T, et al., "Cancer statistics, 2004”, C A Cancer J. Clin. (2004), 54: pp. 8-29.

3. Ferlay J, Bray F, Pisani P and Parkin D M, "GLOBOCAN 2002: Cancer Incidence, Mortality and Prevalence Worldwide", IARC CancerBase No. 5, version 2.0. Lyon, France: IARC Press, Available at: http://wwwdep.iar.fr/globocan/GLOBOframe.htm Accessed November 16, 2004.

4. Goodin S, Rao KV and DiPaola R S, "State-of-the-art treatment of metastatic hormone-refractory prostate cancer", Oncologist (2002), 7: pp. 360-370

5. Coleman R E, "Metastatic bone disease: clinical features, pathophysiology and treatment strategies", Cancer Treat. Rev. (2001), 27: pp. 165-176.

6. Saad F, Gleason D M, Murray $R$ et al., "Long-term efficacy of zoledronic acid for the prevention of skeletal complications in patients with metastatic hormone-refractory prostate cancer", J. Natl. Cancer Inst. (2004), 96: pp. 879-882.

7. Saad F, Gleason D, Murray R et al., "Zoledronic acid is well tolerated for up to 24 months and significantly reduces skeletal complications in patients with advanced prostate cancer metastatic to bone", [poster], Poster presented at: American Urological Association Annual Meeting; April 26 May 1, 2003; Chicago, Illinois. Abstract 1472.

8. Weinfurt K P, LiY, Castel L D, Timbie JW, Glendenning $A$ and Schulman KA, "The impact of skeletal-related events on healthrelated quality of life of patients with metastatic prostate cancer”, [abstract] Ann. Oncol. (2002), 13 (suppl 5): p. 180. Abstract p. 662.

9. Hillner B E, Ingle J N, Chlebowski R T et al., "American Society of Clinical Oncology 2003 update on the role of bisphosphonates and bone health issues in women with breast cancer", [published erratum appears in J. Clin. Oncol. (2004), 22: 1351], J. Clin. Oncol. (2003), 21: pp. 4,042-4,057.

10. Carey P O and Lippert M C, "Treatment of painful prostatic bone metastases with oral etidronate disodium", Urology (1988), 32: pp. 403-407.

11. Kylmälä T, Tammela T L, Lindholm T S and Seppanen J, "The effect of combined intravenous and oral clodronate treatment on bone pain in patients with metastatic prostate cancer", Ann. Chir. Gynaecol. (1994), 83: pp. 316-319.

12. Cresswell $S M$, English $P J$, Hall $R R$, Roberts $J T$ and Marsh $M M$, "Pain relief and quality-of-life assessment following intravenous and oral clodronate in hormone-escaped metastatic prostate cancer”, Br. J. Urol. (1995), 76: pp. 360-365.

13. Heidenreich A, Hofmann $R$ and Engelmann $U H$, "The use of bisphosphonate for the palliative treatment of painful bone metastasis due to hormone refractory prostate cancer", J. Urol. (2001), 165: pp. 136-140.

14. Elomaa I, Kylmälä T, Tammela T, et al., "Effect of oral clodronate on bone pain. A controlled study in patients with metastatic prostatic cancer”, Int. Urol. Nephrol. (1992), 24: pp. 159-166.

15. Kylmälä T, Taube T, Tammela T L, Risteli L, Risteli J and Elomaa I, "Concomitant i.v. and oral clodronate in the relief of bone pain-a double-blind placebo-controlled study in patients with prostate cancer”, Br. J. Cancer (1997), 76: pp. 939-942.

16. Strang P, Nilsson S, Brandstedt S, et al., "The analgesic efficacy of clodronate compared with placebo in patients with painful bone metastases from prostatic cancer”, Anticancer Res. (1997), 17: pp. 4,717-4,721.

17. Ernst D S, Tannock I F, Winquist E W, et al., "Randomized, double-blind, controlled trial of mitoxantrone/prednisone and clodronate versus mitoxantrone/prednisone and placebo in patients with hormone-refractory prostate cancer and pain", J. Clin. Oncol. (2003), 21: pp. 3,335-3,342.

18. Dearnaley D, Sydes M R, Mason M D, et al., "A double-blind, placebo-controlled, randomized trial of oral sodium clodronate for metastatic prostate cancer (MRC PR05 Trial)”, J. Natl. Cancer Inst. (2003), 95: pp. 1,300-1,311.

19. Lipton A, Glover D, Harvey $H$, et al., "Pamidronate in the treatment of bone metastases: results of 2 dose-ranging trials in patients with breast or prostate cancer”, Ann. Oncol. (1994), 5 (suppl 7): pp. S31-S35.

20. Small EJ, Smith M R, Seaman J J, Petrone S and Kowalski M O, "Combined analysis of two multicenter, randomized, placebocontrolled studies of pamidronate disodium for the palliation of bone pain in men with metastatic prostate cancer", J. Clin. Oncol. (2003), 21: pp. 4,277-4,284. 
21. Heidenreich A, Elert $A$ and Hofmann R, "Ibandronate in the treatment of prostate cancer associated painful osseous metastases", Prostate Cancer Prostatic Dis. (2002), 5: pp. 231-235.

22. Saad F, Gleason D M, Murray R, et al., "A randomized, placebo-controlled trial of zoledronic acid in patients with hormonerefractory metastatic prostate carcinoma”, For the Zoledronic Acid Prostate Cancer Study Group, J. Nat. Cancer Inst. (2002), 94: pp. 1,458-1,468.

23. Major P P and Cook R, "Efficacy of bisphosphonates in the management of skeletal complications of bone metastases and selection of clinical endpoints”, Am. J. Clin. Oncol. (2002), 25 (suppl 1): pp. S10-S18.

24. Lipton A, Small E, Saad F, et al., "The new bisphosphonate, Zometa ( $r$ ) (zoledronic acid), decreases skeletal complications in both osteolytic and osteoblastic lesions: a comparison to pamidronate”, Cancer Invest. (2002), 20 (suppl 2): pp. 45-54.

25. Saad F, Gleason D, Murray R, et al., "Early and sustained clinical benefit of zoledronic acid in men with advanced prostate cancer (PC) metastatic to bone: comparison with pamidronate", [poster], Poster presented at: Canadian Urological Association 59th Annual Meeting; June 27 July 1, 2004; Whistler, British Columbia. Abstract 30.

26. Gleason D, Saad F, Goas $A$ and Zheng M, "Continuing benefit of zoledronic acid in preventing skeletal complications after the first occurrence in patients with prostate cancer and bone metastases", [abstract], Proc. Am. Soc. Clin. Oncol. (2003), 22: p. 379, Abstract 1522.

27. Saad F, Gleason D, Murray R, et al., "Long-term reduction of bone pain with zoledronic acid in patients with advanced prostate cancer metastatic to bone”, [poster], Poster presented at:American Urological Association Annual Meeting; April 26 May 1, 2003; Chicago, Illinois, Abstract 1473.

28. Major P P, Cook R J, Chen B-L and Zheng $M$, "Zoledronic acid reduces the need for radiation to bone in patients with breast or prostate cancer metastatic to bone: a survival-adjusted cumulative incidence analysis”, [abstract], Proc. Am. Soc. Clin. Oncol. (2004), 23: p. 739, Abstract 8058.

29. Carroll P R, Altwein J, Brawley O, et al., "Management of disseminated prostate cancer", In: Denis L, Bartsch G, Khoury S, et al, (eds), Prostate Cancer: 3rd International Consultation on Prostate Cancer-Paris. Paris: Health Publications; (2003), pp. 251-284.

30. Smith M R, Eastham J, Gleason D M, Shasha D, Tchekmedyian S and Zinner N, "Randomized controlled trial of zoledronic acid to prevent bone loss in men receiving androgen deprivation therapy for nonmetastatic prostate cancer", J. Urol. (2003), 169: pp. 2,008-2,012.

31. Padalecki S S, Carreon M, Grubbs B, Cui $Y$ and Guise T A, "Androgen deprivation enhances bone loss and prostate cancer metastases to bone: prevention by zoledronic acid”, Oncology (2003), 17 (suppl 3): p. 32. 